\title{
Clustering of heavy particles in vortical flows: a selective review
}

\author{
S RAVICHANDRAN*(i), P DEEPU and RAMA GOVINDARAJAN
}

TIFR Centre for Interdisciplinary Sciences, Hyderabad 500075, India

e-mail: ravis@tifrh.res.in

MS received 21 June 2016; revised 16 August 2016; accepted 4 October 2016

\begin{abstract}
Heavy particles in a turbulent flow tend to leave regions of high vorticity and cluster into regions of high strain. The consequences of such clustering have been studied in a variety of situations over the past few decades, and this problem has seen several review papers already. Our objectives in this paper are three-fold. (i) We introduce the reader to the basic ideas, and explain why the problem is interesting. (ii) Using an $N$-vortex system we present an interesting case where particles are attracted to the vicinity of vortices. A new scaling for the critical Stokes number of attraction is obtained. (iii) We review a number of papers, which are related to cloud physics in this context.
\end{abstract}

Keywords. Vortical flows; inertial particles; preferential clustering; sling caustics; particle collisions; warm rain initiation.

\section{Introduction and outline}

When we add milk to tea and stir, we get a homogeneous distribution of milk in tea. But when particles of larger density than the fluid are added into a turbulent flow, they cluster preferentially into some regions and their number density in the fluid is not uniform. This is a well-known phenomenon seen in both experiments (see [1] for the classical experiment, and [2-4] for more recent experiments) and numerical simulations (see [5] for the earliest simulation and [6-13] for more recent work). This feature is of great interest since turbulent flows laden with particles are ubiquitous in nature and in industrial applications. Dust storms, sand dunes, snow avalanches, ocean spray, sediment flows and plankton blooms (see e.g. [14, 15]) are just some of the situations of interest.

Decades of research has therefore gone into this area, and several review articles have been written on it (in particular [16-22]). We do not therefore attempt an exhaustive review of the vast literature on particle clustering. We will focus on the dynamics of small heavy inertial particles (in which the density of the particle $\rho_{p}$ is much greater than the density of the fluid $\rho_{f}$ ). We are motivated primarily by the dynamics of inertial particles and their relationship to rain formation in clouds; the studies we review will, therefore, be primarily of interest to researchers interested in cloud physics. We have tried to make the paper self-contained.

We restrict ourselves to dilute particle suspensions in incompressible flow. Also the particles of our interest are small enough that the Reynolds number based on the particle radius and relative velocity with the fluid are very small, so the fluid applies Stokes drag on the particle. A one-way coupling, in which the fluid flow determines the particle dynamics, but the particles do not affect the flow, is a fair assumption in this case. The main parameter in the problem, therefore, apart from the flow Reynolds number, is the particle Stokes number $S t \equiv \tau / \tau_{f}$, where $\tau=\frac{2}{9} \frac{a^{2}}{v} \frac{\rho_{p}}{\rho_{f}}$ is the Stokes time-lag associated with the particle, $\tau_{f}$ is a typical flow time-scale, $a$ is the particle radius and $v$ is the kinematic viscosity of the surrounding fluid. Defining $U$ and $L$ as typical flow velocity and length scales, respectively, we have $\tau_{f}=L / U$ and the flow Reynolds number $R e=U L / v$. The Stokes number may be rewritten as

$$
S t=\frac{2}{9} \frac{\epsilon^{2} R e}{R},
$$

where we define $\epsilon \equiv a / L$ as the ratio of particle size to flow length scale, and $R \equiv \rho_{f} / \rho_{p}$, the ratio of fluid and particle densities. For the flows of our interest, $\epsilon \ll 1, R \ll 1$ and $\operatorname{Re} \gg 1$.

Droplets of one liquid in another can also be treated as particles in flow, so long as the droplet Reynolds number is much smaller than unity and the Bond number (which characterises the strength of surface tension) is low. Such droplets will remain spherical. Further, if the droplet viscosity is much higher than the viscosity of the surroundings, then Stokes drag is applicable. In this article we will use the terms 'particle' and 'droplet' interchangeably, but what distinguishes droplets from particles is that when droplets collide with each other, they can coalesce.

*For correspondence 
In section 2, we describe the basic methods used to analyse and study the dynamics of small heavy particles suspended in turbulent flows; in section 3, we describe recent studies from a variety of sub-fields that use the formulation we have described in order to understand physical phenomena.

\section{Heavy particles in turbulence}

The equation of motion for a small sphere in fluid flow was first proposed by Maxey and Riley [23]. If we include the lift force due to Saffman [24], we have the following equation:

$$
\begin{aligned}
\rho_{p} \dot{\mathbf{v}}= & \rho_{f} \frac{D \mathbf{u}}{D t}+\left(\rho_{p}-\rho_{f}\right) \mathbf{g}-\frac{9 v \rho_{f}}{2 a^{2}}\left(\mathbf{v}-\mathbf{u}-\frac{a^{2}}{6} \Delta \mathbf{u}\right) \\
& -\frac{\rho_{f}}{2}\left[\dot{\mathbf{v}}-\frac{D}{D t}\left(\mathbf{u}+\frac{a^{2}}{10} \Delta \mathbf{u}\right)\right] \\
- & \frac{9 \rho_{f}}{2 a} \sqrt{\frac{v}{\pi}} \int_{0}^{t} \frac{d s}{\sqrt{t-s}}\left[\dot{\mathbf{v}}(s)-\frac{d}{d s}\left(\mathbf{u}+\frac{a^{2}}{6} \Delta u\right)_{\mathbf{x}=\mathbf{x}(s)}\right] \\
& +\frac{6.46 \rho_{f}}{\frac{4}{3} \pi a} \sqrt{\frac{v}{|\omega|}}((\mathbf{v}-\mathbf{u}) \times \omega),
\end{aligned}
$$

where $\mathbf{v}$ and $\mathbf{u}$ are the particle and fluid velocities, respectively, $\omega$ is the local vorticity, $\Delta$ is the Laplacian operator, $\mathbf{g}$ is the acceleration due to gravity, $D / D t$ is the material derivative following a fluid streamline and an overdot represents a derivative in time following a particle's trajectory. In order, the right hand side of Eq. (2.1) has the following terms: inertial force, buoyancy, Stokes drag, the added mass, which accounts for the fact that the particle imparts kinetic energy to the surrounding fluid, the Basset history, which accounts for the wake left by the particle along its path and the Saffman lift term, which is analogous to a Magnus force. The terms with a factor $a^{2} \Delta u$ are called Faxen corrections, and account for changes in the flow over length scales of the particle size.

Equation (2.1) in nondimensional form reads

$$
\begin{aligned}
\dot{\mathbf{v}} & =R \frac{D \mathbf{u}}{D t}+\frac{1-R}{F r}-\frac{1}{S t}\left(\mathbf{v}-\mathbf{u}-\frac{\epsilon^{2}}{6} \Delta \mathbf{u}\right) \\
& -\frac{R}{2}\left[\dot{\mathbf{v}}-\frac{D}{D t}\left(\mathbf{u}+\frac{\epsilon^{2}}{10} \Delta \mathbf{u}\right)\right] \\
& -\sqrt{\frac{9 R}{2 \pi S t}} \int_{0}^{t} \frac{d s}{\sqrt{t-s}}\left[\dot{\mathbf{v}}(s)-\frac{d}{d s}\left(\mathbf{u}+\frac{\epsilon^{2}}{6} \Delta u\right)_{\mathbf{x}=\mathbf{x}(s)}\right] \\
& +\frac{10.28}{\pi} \sqrt{\frac{R}{S t|\omega|}}((\mathbf{v}-\mathbf{u}) \times \omega)
\end{aligned}
$$

The Froude number $F r \equiv U^{2} / g L$ gives the ratio of inertial to gravitation forces in the flow. Therefore, in the small heavy particle $(\epsilon \ll 1, R \ll 1)$ limit, if we additionally impose $R \ll S t$ and $F r \gg 1$ the equation governing the motion of the particle reduces to

$$
\begin{aligned}
& \frac{d \mathbf{x}}{d t}=\mathbf{v} \\
& \frac{d \mathbf{v}}{d t}=\frac{\mathbf{u}-\mathbf{v}}{S t} .
\end{aligned}
$$

Several fundamental properties of the dynamics of such particles are known. In particular, the dissipative nature of the particle dynamics results in the particles occupying an evershrinking region of phase space. This can be seen by noting that the rate of contraction of the volume $V$ of phase space accessible to the dynamical system given by Eq. (2.3) is $\frac{\partial V}{\partial t}=$ $\nabla \cdot J=-N_{d} / S t$ in $N_{d}$-dimensional space. Here, $J$ is the current given by the 4 component vector $[\mathbf{v},(\mathbf{u}-\mathbf{v}) / S t]^{T}$, and the operator $\nabla$ consists of partial derivatives with respect to the phase space coordinates, i.e., components of $\mathbf{x}$ and $\mathbf{v}$. Even the region of physical space occupied by the particles shrinks. Bec [6] showed for randomly stirred flows that the space occupied by the particles is a fractal attractor.

Thus we may obtain complex particle dynamics with even a simple linear Stokes drag model, where the drag is proportional to the relative velocity between particle and fluid (2.3). Many simplifications are adopted to derive this model as mentioned earlier. As long as the particle Reynolds number is $\lesssim 0.01$ [25], and the particle radius is much smaller than the Kolmogorov scale, this is a good approximation. Direct Numerical Simulations (DNS) of turbulence-particle interaction based on the linear Stokes drag model are shown to accurately capture the experimentally observed spatial clustering of inertial particles in isotropic turbulence [11]. The assumption of linear drag fails for particles with large $S t(\gtrsim 3)$ settling in gravity, where nonlinear effects are required to reproduce the experimentally observed behaviour that large particles settle slower in turbulent flow than in a quiescent fluid [26]. However, since most studies assume a linear drag, we will limit our discussion to the linear drag model of Eq. (2.3).

It is obvious that the time scale on which the velocity of the particle adjusts to the flow time scale is very different from the time scale associated with the motion of the particle itself. Haller and Sapsis [27] show that Eq. (2.3) can be treated as a singular perturbation problem in the Stokes time scale, and that the leading term in the perturbation expansion may be found by rearranging (2.3) to give

$$
\begin{gathered}
\mathbf{v}=\mathbf{u}-S t \frac{d \mathbf{v}}{d t}, \\
\text { i.e., } \quad \mathbf{v}=\mathbf{u}-S t \frac{d\{\mathbf{u}-S t d \mathbf{v} / d t]\}}{d t} .
\end{gathered}
$$


It is standard practice to neglect higher powers of the Stokes number at small Stokes number. Thus, for small enough Stokes number, the motion of the particles is approximated by the so-called inertial equation

$$
\mathbf{v}=\mathbf{u}-S t \frac{D \mathbf{u}}{D t} .
$$

Note that, crucially, Eq. (2.6) describes the motion of particles in terms of a velocity field, whereas Eq. (2.3) does not. The consequence of this is that Eq. (2.6) fails to account for caustics (see section 2.3). This approximation, however, is useful in the small Stokes limit to explain the dynamics, as done later.

\subsection{Heavy particles are expelled from vortical regions}

A direct consequence of Eq. (2.3) is that heavy particles are expelled out of regions of high vorticity and settle in regions of high strain. To see this clearly, we write Eq. (2.3) in cylindrical polar $(r-\theta)$ coordinates, and model the region of high vorticity as a point vortex at the origin (so that the fluid velocity is $\mathbf{u}_{f}=\frac{\Gamma}{2 \pi} \frac{\mathbf{e}_{\theta}}{r}$ ). The location of a particle is its radius vector, $\mathbf{r}=r \mathbf{e}_{r}$. The velocity and acceleration of the particle are therefore

$$
\begin{aligned}
\mathbf{v} & =\frac{d \mathbf{r}}{d t}=\mathbf{e}_{r} \frac{d r}{d t}+\mathbf{e}_{\theta} r \frac{d \theta}{d t} \\
\frac{d \mathbf{v}}{d t} & =\mathbf{e}_{r} \frac{d^{2} r}{d t^{2}}+2 \mathbf{e}_{\theta} \frac{d r}{d t} \frac{d \theta}{d t}-\mathbf{e}_{r} r\left(\frac{d \theta}{d t}\right)^{2}+\mathbf{e}_{\theta} r \frac{d^{2} \theta}{d t^{2}} .
\end{aligned}
$$

Writing these equations along the coordinate directions, we get

$$
\begin{aligned}
\frac{d r}{d t} & =v_{r} \\
\frac{d \theta}{d t} & =\omega \\
\frac{d v_{r}}{d t} & =-\frac{v_{r}}{\tau}+r \omega^{2} \\
\frac{d \omega}{d t} & =\frac{\Omega-\omega}{\tau}-2 \frac{v_{r}}{r} \omega .
\end{aligned}
$$

Here $\Omega$ is the vorticity in the flow, $r$ and $\theta$ are the radial and azimuthal position of the particle measured from a given origin, $v_{r}$ the radial velocity and $\omega$ the angular velocity of the particle.

If we assume an axisymmetric velocity field, such as that of a vortex, and that the particle has an initial velocity equal to that of the fluid, Eq. (2.7) can be used to show that the particle a) maintains the same angular velocity as the fluid; and b) drifts outwards with a velocity $v_{r}$ that obeys the third of Eq. (2.7) (see [28]). It follows that all heavy particles are expelled outwards from the centre of a vortex. Another way to see this is as follows.
The flows we consider are incompressible, i.e. $\nabla \cdot \mathbf{u}=0$. However, since particles cluster into regions of high number density, the particle velocity field $\mathbf{v}$ will not be divergence-free. In regions from where particles are expelled, we must have $\nabla \cdot \mathbf{v}>0$, and in regions where they collect, we must have $\nabla \cdot \mathbf{v}<0$. We may take the divergence of the inertial equation (2.6), and use the fact that $\nabla \cdot \mathbf{u}=0$ to get the divergence of the particle velocity as

$$
\nabla \cdot \mathbf{v}=-\tau \nabla \cdot \frac{D \mathbf{u}}{D t} .
$$

This gives, since $D \mathbf{u} / D t=\partial \mathbf{u} / \partial t+\mathbf{u} \cdot \nabla \mathbf{u} \quad$ and $\nabla \cdot \partial \mathbf{u} / \partial t=0$

$$
\nabla \cdot \mathbf{v}=-\tau \nabla \cdot(\mathbf{u} \cdot \nabla \mathbf{u}) .
$$

This can be expanded to (in index notation; note that we have switched to Cartesian coordinates here, but the identity will not change)

$$
\nabla \cdot(\mathbf{u} \cdot \nabla \mathbf{u})=\frac{\partial}{\partial x_{i}}\left(u_{j} \frac{\partial u_{i}}{\partial x_{j}}\right)=\frac{\partial u_{i}}{\partial x_{j}} \frac{\partial u_{j}}{\partial x_{i}} .
$$

We then note that

$$
\frac{\partial u_{i}}{\partial x_{j}}=S_{i j}+\Omega_{i j}
$$

where $S_{i j}=\frac{1}{2}\left(\frac{\partial u_{i}}{\partial x_{j}}+\frac{\partial u_{j}}{\partial x_{i}}\right)$ and $\Omega_{i j}=\frac{1}{2}\left(\frac{\partial u_{i}}{\partial x_{j}}-\frac{\partial u_{j}}{\partial x_{i}}\right)$ are respectively, the symmetric and anti-symmetric parts of the velocity gradient tensor, and this gives

$$
\nabla \cdot \mathbf{v}=-\tau\left(S^{2}-\Omega^{2}\right)=\tau Q .
$$

The quantity $Q=\left(\Omega^{2}-S^{2}\right)$ is called the Okubo-Weiss parameter, and predicts where particles in a flow will end up. Using the Okubo-Weiss condition, several things can be predicted about particle clustering. It is evident from Eq. (2.8) that particles leave regions where $\Omega>S$ and cluster into regions where $S>\Omega$, i.e., a negative value for the $Q$ is a necessary condition for particles to cluster. In fact, Haller and Sapsis [27] show, for a fixed frame of reference, that the Okubo-Weiss parameter cannot be negative in a region with closed streamlines. This can be pictured as saying that heavy particles cannot cluster near a vortex, as shown schematically in figure 1 .

The simple picture given here no longer holds in rotating frames (as shown in [29]), leading to the following interesting consequences.

\subsection{Attracting fixed points in rotating frames}

In a rotating frame, there can be attracting fixed points in the vicinity of vortices, where a large number of particles can cluster. In particular, these fixed points are within closed streamlines in the rotating frame of reference. In 


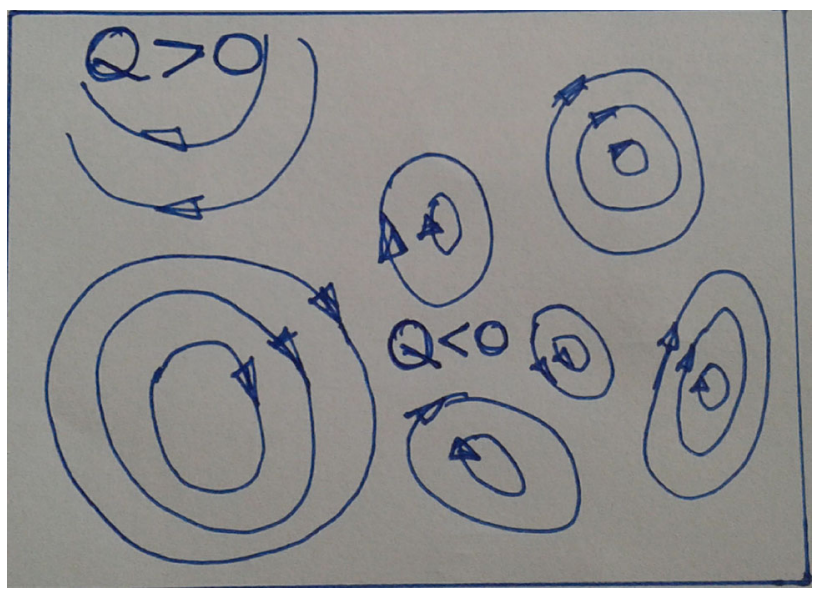

Figure 1. Vortical regions with closed streamlines, where $Q>0$, and strain-dominated regions, where $Q<0$. In steady flow, heavy particles are always centrifuged out of vortical regions and collect in strain-dominated regions.

two-dimensional turbulence, vortices often group themselves into twos and threes and these interactions can result in 'non-traditional' clustering of particles [29], as shown below.

Consider two identical like-signed point vortices rotating about each other. Given the strength of the vortices, the angular velocity with which they will rotate about a point midway between them is a known constant. In a frame of reference centred at this point and rotating at this angular velocity, therefore, the two vortices are stationary. While we may expect that what is true of one vortex (that heavy particles leave the vortex) is doubly true of a pair of vortices, we find that there exist, in fact, stable attracting fixed points at which the particles can aggregate as long as the vortices exist. The fixed points are found by solving the pair of equations (where $\Omega$ is the angular velocity with which the frame rotates)

$$
\begin{aligned}
& \frac{d \hat{\mathbf{x}}}{d \hat{t}}=\hat{\mathbf{v}} \\
& \frac{d \hat{\mathbf{v}}}{d \hat{t}}=\frac{\hat{\mathbf{u}}-\hat{\mathbf{v}}}{S t}-2 \vec{\Omega} \times \hat{\mathbf{v}}+\Omega^{2} \hat{\mathbf{r}} .
\end{aligned}
$$

Note that these are 'fixed' points only in the rotating frame of reference. In the lab-fixed frame, these points execute limit cycles. These fixed points are attractive to small heavy particles of Stokes number below a critical value. The location of these fixed points is dependent on Stokes number as well. A very high number of particles arrive at these fixed points, and co-rotate with them. Fixed points for heavy inertial particles of this kind can also be found for regular arrangements of $N>2$ vortices. Figure 2 shows the basin boundaries for the elliptic fixed points that occur next to four like-signed vortices. In this figure there are four fixed points close to the tips of the spiral seen. All particles of Stokes number 1/300 that

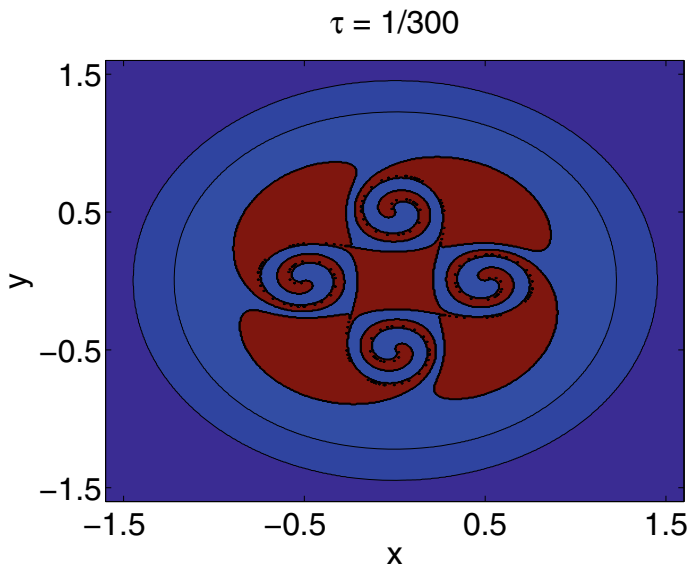

Figure 2. The basins of attraction of the elliptic fixed points for a system of four identical like-signed vortices are shown in red.

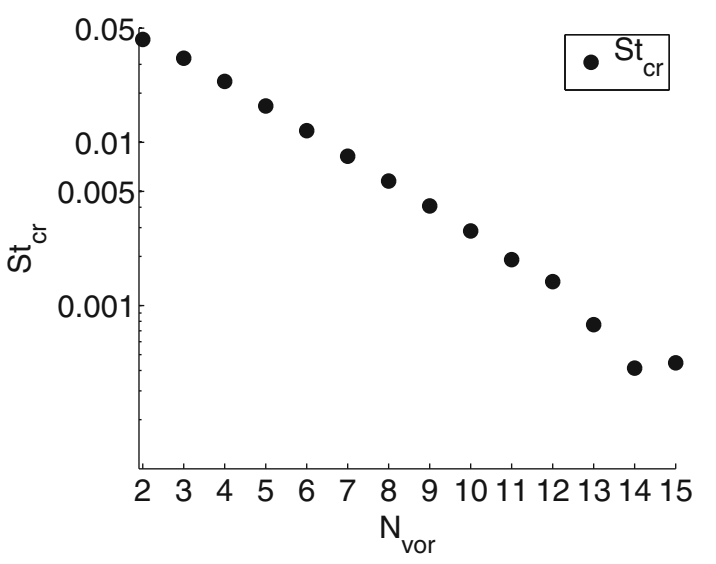

Figure 3. The critical Stokes number for any number of vortices. Particles below the critical Stokes number cluster into fixed points in the vicinity of the vortices.

start in the red regions settle into one of the four attracting fixed points. Given that the basin of attraction extends over a significant area, it is easy to imagine that the number density of particles is extremely high at the fixed points.

Similarly, in an $N$-vortex configuration, when $N$ identical point vortices are placed at the vertices of a regular polygon, we have $N$ fixed points. The behaviour of $N$ vortices started out thus is interesting in itself, and the reader is referred to [30]. In the present article we ask how stable the fixed points are, i.e., up to what Stokes number particles can they attract to themselves. Figure 3 shows that the critical Stokes number falls exponentially with the number of vortices. As mentioned earlier, normally vortices interact in smaller groups; hence these results suggest that moving fixed points need to be studied further as a mechanism for producing enormous particle clustering into extremely small neighbourhoods. 


\subsection{Caustics}

We noted that expressing the particle velocity as a field and expressing its divergence in terms of the Okubo-Weiss parameter is an approximation at $\mathcal{O}(S t)$. In general, however, the velocities of inertial particles in fluid flow do not form a field, and can be multi-valued. That is, two particles can exist at the same location at a given time with (very) different velocities. This phenomenon is called caustics, and has been proposed independently by Mehlig and Wilkinson and coworkers [31] and by Falkovich and coworkers [32] and is beginning to be observed in experiments (see [33]). Caustics are known to play an important role in the clustering of inertial particles for larger Stokes numbers. This is usually understood as being because particles of small Stokes numbers are much more susceptible to preferential concentration by coherent structures, while, as the inertia increases, the role of 'ergodic' or noncoherent flow structures becomes ever more important (see [34]) and the clustering of particles depends on particle histories. We now discuss the literature on caustics and other relevant aspects of particle clustering, with most of our attention given to the initiation of rain in clouds.

\section{Clustering, collisions and droplet growth in a cloud}

As mentioned, our interest is mainly in the applications of the theory of Stokesian particles to water droplets in clouds. We are interested, in particular, in warm cumulus clouds ('warm' because these clouds have no ice, and 'cumulus' because they appear like heaps and have significant turbulence). Cumulus clouds that are capable of bearing rain have relatively short lifetimes-they go from being essentially large parcels of moist air to clouds that rain in half an hour or less. No complete explanation has been found for this rapidity of onset of rain.

Clouds start with condensation nuclei (salt or dust particles) that are fractions of a micron in diameter. These nuclei initially grow by vapour diffusion in the supersaturated environment afforded to them by the cloud. This rate of growth by vapour diffusion is inversely proportional to the particle radius; for particles larger than $10-20 \mu \mathrm{m}$, the rate of growth is much too small. At the other end of the droplet size range, droplets that are about $50 \mu \mathrm{m}$ or larger have significant rates of collision with other droplets (either of their own size or with smaller droplets), and can grow relatively quickly. The problem of explaining how droplets grow from about 10 to about $50 \mu \mathrm{m}$ on time scales short enough to explain rapid rain formation is a long-unsolved problem of much interest.

The first attempts at explaining the 'droplet growth bottleneck' invoked only the fact that inertial particles preferentially sample regions of the flow with low vorticity or high strain. Shaw et al [12] argued that the regions of the flow with high vorticity would also end up with greater supersaturations than high-strain regions, and therefore be able to nucleate and grow particles much faster. Several objections were raised against this prediction, including by Vaillancourt, Yau, Grabowski and co-workers [13, 21, 35], who point out in the latter two papers that the decoherence times fall as the Reynolds numbers increase, meaning that particles that may have sampled a high supersaturation region at one instant will soon sample a region with lower supersaturation (see also the review by Grabowski and Wang [17]). In short, preferential concentration by itself is not enough to explain the droplet size broadening observed in clouds. Therefore, it cannot, by itself, explain why rain initiation times are short. However, preferentially concentrated droplets are, it may be imagined, prone to larger rates of collision, and if enough collisions occur, particles can quickly grow in size. Preferential concentration remains of indirect interest, therefore.

In addition to clustering, inertial particles in turbulent flows also have higher rates of collision than that for particles that follow fluid streamlines ('fluid particles'), first derived by Saffman and Turner [36] (see Eq. (3.3)). The first numerical study of collision rates was done by Sundaram and Collins [37], followed by a large number of other studies. The phenomenon of caustics (section 2.3), which is thought to account for the increased rate of collisions, is the focus of much research on turbulent particleladen flows (see, e.g. [38-42]). The line of reasoning followed by such studies is the following.

Consider a reference particle relative to which the rest of the flow and the particles move. If all $n$ particles per unit volume in the flow are of the same radius $a$, the collision zone of the reference particle is a sphere of radius $2 a$ centred on it. Hence $n 4 \pi(2 a)^{2}\left\langle\left|w_{r}\right|\right\rangle$ is the number of particles coming into its collision zone per unit time. The number of particles colliding with our reference particle per unit time is, then,

$$
\mathcal{R}=\frac{1}{2} 4 \pi n(2 a)^{2}\left\langle\left|w_{r}\right|\right\rangle=n \Gamma,
$$

where $\left\langle\left|w_{r}\right|\right\rangle$ denotes the average magnitude of the radial component of the velocity of particles relative to the reference particle. The factor $\frac{1}{2}$ accounts for the fact that, in an incompressible particulate flow, only half of the particles on an average are approaching the target particle, and the other half is moving away. This way of finding the collision rate-using a sphere centred on the reference particle-is called the spherical volume formulation, and, in turbulent flows, is found to predict collision rates better than the more familiar cylindrical formulation in which the reference particle is assumed to trace out a cylinder of radius $2 a$. For molecules of a gas, the two formulations are identical. For details, see [43].

Here $\Gamma$ is called the collision kernel and its definition is clear from the above equation. Since we are interested in 
the growth of droplet size, we should look at the rate of coalescence, which can be obtained by multiplying the collision rate by a coalescence efficiency $E$ to get

$$
\mathcal{R}=n E \Gamma=n \kappa,
$$

where $\kappa$ is termed as the collection kernel. The coalescence efficiency is the fraction of collision events resulting in droplet coalescence. Due to the lubricating layer trapped between the approaching particles, among other reasons [44], all collisions need not result in coalescence. But in the case of colliding cloud droplets, it is shown that coalescence efficiency is $\approx 1$ [45] and in the following discussion, we consider $100 \%$ coalescence efficiency. Under this approximation, collision rate is the same as coalescence rate.

In the limit of $S t \rightarrow 0$, the particles follow the fluid streamlines exactly and the collision occurs only due to shearing action; hence, $\left\langle\left|w_{r}\right|\right\rangle \sim 2 a / \tau_{K}$, where $\tau_{K}$ is the collision time scale. Saffman and Turner [36] derived the following exact expression for the collision rate of tracers suspended in isotropic turbulence and approximating the velocity field around the reference particle as hyperbolic and the velocity gradients to be Gaussian-distributed;

$$
\mathcal{R}_{S T}=\sqrt{\frac{8 \pi}{15}} \frac{n(2 a)^{3}}{\tau_{K}} .
$$

Note that this formula does not take into account the effect of the concentration inhomogeneity due to preferential clustering because the velocity field of suspended tracers is divergence free. The effect of preferential concentration at higher $S t$ is incorporated by changing the particle density at a distance $r$ from the reference particle to $n g(r)$, where $g(r)$ is a radial correlation function and is defined as the ratio of probability density of finding a pair of particle at distance $r$ in the real system to that in a system with uniform distribution of particles. Hence, for uniform concentration, $g=1$. For a detailed comparison of different theoretical models describing the spatial distribution and the relative velocities of inertial particles in isotropic turbulence, see refs. [34, 46]. If it is assumed that particle clustering does not significantly change the relative velocity, the rate of collision becomes

$$
\mathcal{R}_{a d v}=\sqrt{\frac{8 \pi}{15}} \frac{n(2 a)^{3}}{\tau_{K}} g(2 a)
$$

At a given St, $g(r)$ exhibits a power-law dependence on $r$ : $g(r) \sim r^{-\varsigma}$ [47] and $0<\varsigma<0.7$ [7]. However, Sundaram and Collins [37] reported an abrupt increase in collision rate in their numerical experiments, as the Stokes number exceeds a threshold value. Preferential clustering cannot explain this observation, as it is thought to be effective only when the particle relaxation time matches the correlation time of the fluid flow field, or, $S t \sim 1$. The phenomenon of caustics (also called "sling caustics" or "fold caustics"), briefly described in section 2.3, was proposed as the explanation. Caustics occur when there are large negative velocity gradients in the flow, causing the momentum-position manifold in the phase space of the particles to fold over itself. As a result the particle velocity field becomes multi-valued, leading to the existence of particles with large variation in velocity within a small separation. In addition to this effect, caustics also leads to a non-local clustering mechanism at high $S t$, whereby the particle exhibits a memory of its interaction with the fluid velocity in its path history [48, 49].

At very large Stokes number, the particles become completely uncorrelated with the fluid motion and hence their motion can be described by gas-kinetic theory [50]. Subsequently, $\left\langle\left|w_{r}\right|\right\rangle \sim \frac{\eta}{\tau_{K}} F(S t, R e)$, where the function $F(S t, R e)$ represents the rate of caustic formation [51]. Hence the rate of collision due to caustics is given by

$$
\mathcal{R}_{\text {caust }}=\frac{n a^{2} \eta}{\tau_{K}} F(S t, R e) .
$$

The total collision rate is approximated by the sum of the contributions due to preferential concentration $\left(\mathcal{R}_{a d v}\right)$ and caustics $\left(\mathcal{R}_{\text {caust }}\right)$ [42]:

$$
\mathcal{R}=\mathcal{R}_{\text {adv }}+\mathcal{R}_{\text {caust }} .
$$

In the limit of $S t \rightarrow 0$, the function $F$ has the asymptotic form of $F(S t, R e) \sim \exp (-C / S t)$. This is obtained from a $1-\mathrm{D}$ white noise model and is motivated by the notion that caustics formation has an activated form similar to the Arrhenius term determining the rate of an activated chemical process [31]. Numerical simulations of NavierStokes equations [38] show that the action $C \approx 2$. Note that as $S t \rightarrow 0, \quad F \rightarrow 0$, thus reducing Eq. (3.6) to Eq. (3.3). In the opposite limit of large $S t, F(S t, \infty) \sim$ $K \sqrt{S t}$ because at large $R e$, the relative velocity can depend only on the turbulent energy dissipation rate $\epsilon$ and the particle response time $\tau_{P}$. Dimensional analysis gives $\left\langle\left|w_{r}\right|\right\rangle \sim \sqrt{\epsilon \tau_{P}}$ [39]. Flows modelled using kinematic simulations (in which the fluid velocity field is described by a random field tailored in such a way that its statistics follows the Kolmogorov theory for fully developed turbulence) show that $K \approx 50$ [41].

The two central questions to ask, therefore, are the following: first, do caustics explain the enhanced collision rates observed in turbulent flows? and second, does the increased rate of collisions explain the rapid initiation of rain in warm cumulus clouds? There does not seem to be a clear consensus on either question, although we lean towards answering yes to both questions.

Initial work on caustics suggested that the drastic increase in collision rate can explain the rapid onset of rain. However, as we have seen above, caustics were thought to be dominant only for $S t \gtrsim 1$ ([40]), and the typical Stokes numbers of $S t \sim 0.01$ for water droplets in a cloud could 
mean that the influence of caustics is negligible in warm rain initiation [52]. In fact, Wilkinson even suggests [53] that gravity alone (in the light of large deviation theory and "lucky" droplets) is enough to explain rapid rain initiation, based on the fact that the fraction of cloud droplet that has to undergo a runaway growth is less than one in a trillion. However, as estimated by [52], this theory can explain runaway droplet growth only after the droplet size already reaches $50 \mu \mathrm{m}$.

In addition, Khain et al [18] point out that analytical and numerical studies tend to overestimate the increase in the rate of collisions on account of ignoring important effects like gravitational settling. Recent studies are divided on this question. Bec et al [54] find that gravity has competing effects on the two factors-clustering of particles and relative velocities between particles-that determine the collision rate. Gravity increases the amount of clustering, but decreases the relative velocities between particles. For small $S t$, they report that the increase in clustering dominates the decrease in relative velocities, leading to a net increase in the collision rates. Other studies by Park and Lee [55] find that for $S t>1$ and strong gravity, the clustering of particles increases. Comprehensive studies by Ireland et al $[56,57]$ find that gravitational settling reduces the clustering for small Stokes numbers, while increasing the degree of clustering for larger Stokes numbers. The latter two studies also suggest that the rates of collisions between like-sized particles are significantly lower because of gravity for Stokes numbers $S t \gtrsim 0.1$.

In spite of these findings, the central idea that particle collisions induced by turbulence are an essential part of the explanation of rapid rain initiation holds. This is partly because the Stokes numbers in clouds are, at $S t=\mathcal{O}(0.01)$, smaller than the value at which gravity seems to become a factor. In particular, the fact that the interactions of such small particles are likely most effective with vortices in the dissipative range is a reason to believe that the centrifugal effect is still important.

The intermittent nature of turbulent flows leads to the development of localized high vorticity regions. Recent work by Bec et al [58] shows that intermittency of turbulent mixing leads to an enhanced growth rate of coagulating aggregates in dilute suspensions as in a warm cloud. Intermittency has also been invoked in the experimental study by Bewley et al [33] to argue that caustics will be crucial in clouds. Additionally, in ongoing work by our group, we find that droplets in the size range appropriate to clouds interacting with dissipative range vortices are capable of achieving very high collision rates, and hence that the increase of collision rates due to caustics can be expected to play a major role in droplet growth. However, due to the difficulties associated with experimental and numerical studies simulating the actual conditions prevalent in clouds, conclusive evidence-one way or the other-is yet to come by.

\section{Conclusions}

We have discussed the dynamics of small Stokes number heavy particles in steady flow, and explained why they leave regions of high vorticity and cluster into regions of high strain. We then discuss how this analysis misses 'moving fixed points', i.e., points near a vortex where a very large number of particles can cluster, but which are moving in the lab frame of reference. We have shown here that the critical Stokes number for particle aggregation in these fixed points goes down exponentially with increasing number of interacting vortices. Finally, we have discussed a few important studies on droplet growth in clouds to delineate present knowledge of how droplets are thought to grow.

\section{Acknowledgements}

We would like to thank both anonymous referees whose suggestions helped us correct errors that may have led readers astray, and add several points of discussion, which have improved the review substantially.

\section{References}

[1] Fessler J R, Kulick J D and Eaton J K 1994 Preferential concentration of heavy particles in a turbulent channel flow. Phys. Fluids 6(11): 3742-3749

[2] Bateson C P and Aliseda A 2012 Wind tunnel measurements of the preferential concentration of inertial droplets in homogeneous isotropic turbulence. Exp. Fluids 52(6): 1373-1387

[3] Monchaux R, Bourgoin M and Cartellier A 2012 Analyzing preferential concentration and clustering of inertial particles in turbulence. Int. J. Multiphase Flow 40: 1-18

[4] Obligado M, Missaoui M, Monchaux R, Cartellier A and Bourgoin M 2011 Reynolds number influence on preferential concentration of heavy particles in turbulent flows. J. Phys. Conf. Ser. 318(5): 052015

[5] Squires K D and Eaton J K 1991 Preferential concentration of particles by turbulence. Phys. Fluids A 3(5): 1169-1178

[6] Bec J 2003 Fractal clustering of inertial particles in random flows. Phys. Fluids 15(11): 16-20

[7] Bec J, Biferale L, Cencini M, Lanotte A, Musacchio S and Toschi F 2007 Heavy particle concentration in turbulence at dissipative and inertial scales. Phys. Rev. Lett. 98(8): 084502

[8] Coleman S W and Vassilicos J C 2009 A unified sweep-stick mechanism to explain particle clustering in two- and threedimensional homogeneous, isotropic turbulence. Phys. Fluids, 21(11): 1-10

[9] Fung J C H and Vassilicos J C 2003 Inertial particle segregation by turbulence. Phys. Rev. E - Stat., Nonlin. Soft Matter Phys. 68(4pt2): 046309

[10] Gualtieri P, Picano F and Casciola C M 2009 Anisotropic clustering of inertial particles in homogeneous shear flow. $J$. Fluid Mech. 629: 25-39 
[11] Salazar J P L C, De Jong J, Cao L, Woodward S H, Meng H and Collins L R 2008 Experimental and numerical investigation of inertial particle clustering in isotropic turbulence. $J$. Fluid Mech. 600: 245-256

[12] Shaw R A, Reade W C, Collins L R and Verlinde J 1998 Preferential concentration of cloud droplets by turbulence: effects on the early evolution of cumulus cloud droplet spectra. J. Atmos. Sci. 55: 1965-1976

[13] Vaillancourt P A, Yau M K, Bartello P and Grabowski W W 2002 Microscopic approach to cloud droplet growth by condensation. Part II: turbulence, clustering, and condensational growth. J. Atmos. Sci. 59(24): 3421-3435

[14] Dominguez A, van Aartrijk M, Del Castello L and Clercx H J H 2007 Aggregate formation in 3D turbulent-like flows. In: Geurts B, Clercx H and Uijttewaal W (eds) Particle-laden flow: from geophysical to kolmogorov scales. Springer, Dordrecht, pp. 359-371

[15] Reigada R, Hillary R M, Bees M A, Sancho J M and Sagues F 2003 Plankton blooms induced by turbulent flows. Proc. R. Soc. B: Biol. Sci. 270(1517): 875-880

[16] Devenish B J, Bartello P, Brenguier J L, Collins L R, Grabowski W W, Ijzermans R H A, Malinowski S P, Reeks M W, Vassilicos J C, Wang L P and Warhaft Z 2012 Droplet growth in warm turbulent clouds. Q. J. R. Meteorol. Soc. 138(667): 1401-1429

[17] Grabowski W W and Wang L P 2012 Growth of cloud droplets in a turbulent environment. Annu. Rev. Fluid Mech. 45(1): 293-324

[18] Khain A, Pinsky M, Elperin T, Kleeorin N, Rogachevskii I and Kostinski A 2007 Critical comments to results of investigations of drop collisions in turbulent clouds. Atmos. Res. 86(1): 1-20

[19] Mashayek F and Pandya R V R 2003 Analytical description of particle/ droplet-laden turbulent flows. Prog. Energy Combust. Sci. 29(312): 329-378

[20] Shaw R A 2003 Particle-Turbulence Interactions in Atmospheric Clouds. Annu. Rev. Fluid Mech. 35(1): 183-227

[21] Vaillancourt P A and Yau M K 2000 Review of particleturbulence interactions and consequences for cloud physics. Bull. Am. Meteorol. Soc. 81(2): 285-298

[22] Warhaft Z 2008 Laboratory studies of droplets in turbulence: towards understanding the formation of clouds. Fluid Dyn. Res. 41(1): 011201

[23] Maxey M R and Riley J J 1983 Equation of motion for a small rigid sphere in a nonuniform flow. Phys. Fluids 26(4): 883

[24] Saffman P G 1965 The lift on a small sphere in a shear flow. J. Fluid Mech. 22: 385-400

[25] Wood A M, Hwang W and Eaton J K 2005 Preferential concentration of particles in homogeneous and isotropic turbulence. Int. J. Multiphase Flow 10(31): 1220-1230

[26] Good G H, Ireland P J, Bewley G P, Bodenschatz E, Collins L R and Warhaft Z 2014 Settling regimes of inertial particles in isotropic turbulence. J. Fluid Mech. 759: R3

[27] Haller G and Sapsis T 2008 Where do inertial particles go in fluid flows? Physica D: Nonlin. Phenom. 237(5): 573-583

[28] Ravichandran S and Govindarajan R 2015 Caustics and clustering in the vicinity of a vortex. Phys. Fluids 27(3): 033305

[29] Ravichandran S, Perlekar P and Govindarajan R 2014 Attracting fixed points for heavy particles in the vicinity of a vortex pair. Phys. Fluids 26(1): 013303
[30] Swaminathan R V, Ravichandran S, Perlekar P and Govindarajan R 2016 Dynamics of circular arrangements of vorticity in two dimensions. Phys. Rev. E 94: 013105

[31] Wilkinson M and Mehlig B 2005 Caustics in turbulent aerosols. Europhys. Lett. 71(2): 186

[32] Falkovich G, Fouxon A and Stepanov M G 2002 Acceleration of rain initiation by cloud turbulence. Nature 419(9): 151-154

[33] Bewley G P, Saw E W and Bodenschatz E 2013 Observation of the sling effect. New J. Phys. 15: 083051

[34] Bragg A D and Collins L R 2014 New insights from comparing statistical theories for inertial particles in turbulence: I. Spatial distribution of particles. New J. Phys. 16(5): 055013

[35] Vaillancourt P A, Yau M K and Grabowski W W 2001 Microscopic approach to cloud droplet growth by condensation. Part I: model description and results without turbulence. J. Atmos. Sci. 58(14): 1945-1964

[36] Saffman P G f and Turner J S 1956 On the collision of drops in turbulent clouds. J. Fluid Mech. 1(01): 16-30

[37] Sundaram S and Collins L R 1997 Collision statistics in an isotropic particle-laden turbulent suspension. Part 1. Direct numerical simulations. J. Fluid Mech. 335: 75-109

[38] Falkovich G and Pumir A 2007 Sling effect in collisions of water droplets in turbulent clouds. J. Atmos. Sci. 64: 4497

[39] Mehlig B, Uski V and Wilkinson M 2007 Colliding particles in highly turbulent flows. Phys. Fluids 19(9): 098107

[40] Voßkuhle M, Pumir A, Lévêque E and Wilkinson M 2014 Prevalence of the sling effect for enhancing collision rates in turbulent suspensions. J. Fluid Mech. 749: 841-852

[41] Voßkuhle M, Pumir A, Lévêque E and Wilkinson M 2015 Collision rate for suspensions at large stokes numberscomparing navier-stokes and synthetic turbulence. J. Turbul. 16(1):15-25

[42] Wilkinson M, Mehlig B and Bezuglyy V 2006 Caustic activation of rain showers. Phys. Rev. Lett. 97(4): 048501

[43] Wang L P, Wexler A S and Zhou Y 1998 Statistical mechanical descriptions of turbulent coagulation. Phys. Fluids 10(10): 2647-2651

[44] Kollár L E, Farzaneh M and Karev A R 2005 Modeling droplet collision and coalescence in an icing wind tunnel and the influence of these processes on droplet size distribution. Int. J. Multiphase Flow 31(1): 69-92

[45] Beard K V, Durkee R I and Ochs III H T 2002 Coalescence efficiency measurements for minimally charged cloud drops. J. Atmos. Sci. 59(2): 233-243

[46] Bragg A D and Collins L R 2014 New insights from comparing statistical theories for inertial particles in turbulence: II. Relative velocities. New J. Phys. 16: 055014

[47] Reade W C and Collins L R 2000 Effect of preferential concentration on turbulent collision rates. Phys. Fluids, 12(10): 2530-2540

[48] Bragg A D, Ireland P J and Collins L R 2015 Mechanisms for the clustering of inertial particles in the inertial range of isotropic turbulence. Phys, Rev. E 92(2): 023029

[49] Bragg A D, Ireland P J and Collins L R 2015 On the relationship between the non-local clustering mechanism and preferential concentration. J. Fluid Mech. 780: 327-343

[50] Abrahamson J 1975 Collision rates of small particles in a vigorously turbulent fluid. Chem. Eng. Sci. 30(11): 1371-1379 
[51] Gustavsson K and Mehlig B 2016 Statistical model for collisions and recollisions of inertial particles in mixing flows. Eur. Phys. J. E 39(5): 1-9

[52] Pumir A and Wilkinson M 2016 Collisional aggregation due to turbulence. Annu. Rev. Condens. Matter Phys. 7: 141-170

[53] Wilkinson M 2016 Large deviation analysis of rapid onset of rain showers. Phys. rev. lett. 116(1): 018501

[54] Bec J, Homann H and Ray S S 2014 Gravity-driven enhancement of heavy particle clustering in turbulent flow. Phys. Rev. Lett. 112(18): 184501

[55] Park Y and Lee C 2014 Gravity-driven clustering of inertial particles in turbulence. Phys. Rev. E - Stat. Nonlin. Soft Matter Phys. 89(6): 1-5
[56] Ireland P J, Bragg A D and Collins L R 2016 The effect of reynolds number on inertial particle dynamics in isotropic turbulence. Part 1. Simulations without gravitational effects. J. Fluid Mech. 796: 617-658

[57] Ireland P J, Bragg A D and Collins L R 2016 The effect of reynolds number on inertial particle dynamics in isotropic turbulence. Part 2. Simulations with gravitational effects. $J$. Fluid Mech. 796: 659-711, 6

[58] Bec J, Ray S S, Saw E W and Homann H 2016 Abrupt growth of large aggregates by correlated coalescences in turbulent flow. Phys. Rev. E 93(3): 031102 